\title{
Cold-Blooded Cognition: New Directions in Reptile Cognition
}

\section{Satoko Matsubara ${ }^{1}$, D. Charles Deeming ${ }^{2}$ \& Anna Wilkinson ${ }^{2,3}$}

${ }^{1}$ Department of Animal Science and Biotechnology, Azabu University, Kanagawa, Japan.

${ }^{2}$ School of Life Sciences, University of Lincoln, Lincoln, UK

${ }^{3}$ Wildlife Research Center, Kyoto University, 2-24 Tanaka-Sekiden-cho, Sakyo, Kyoto, 606-8203, Japan

Corresponding author:
Anna Wilkinson

School of Life Sciences

University of Lincoln

Lincoln, UK

LN6 7DL

awilkinson@lincoln.ac.uk 


\begin{abstract}
The field of comparative psychology has traditionally focussed on investigating the cognitive abilities of a small number of mammal and bird species, but in order to understand the evolution of cognition, it is essential to examine cognitive abilities across a large range of vertebrates. Reptiles are particularly interesting in this context as they represent a key amniotic Class that do not develop under high, stable temperatures, which can produce phenotypic variation in the population. As their patterns of development differ substantially from those of birds and mammals reptiles can be used to investigate fundamental questions relating to factors shaping cognition; questions that cannot be asked in mammals and birds. In this review, we highlight some of these areas of interest and consider how the emerging field of reptile cognition can address crucial questions in cognitive science.
\end{abstract}




\section{Why study reptile cognition?}

In order to understand the evolution of cognition, it is essential to compare abilities across the animal kingdom. Similarities and differences in performance of the same task, alongside studying the structure of the brain, can inform us about evolution of various cognitive abilities. Traditionally, comparative psychology has focussed a lot of attention on a very few species of mammals and birds [1] and although the number and diversity of species studied has been increasing, the bias towards mammals and birds remains [2]. This work has provided strong evidence that similar, potentially complex, cognitive abilities (e.g. tool use) can be shared among distantly related species [3] whose evolutionary history and brain structures differ substantially [4]. Reptiles are a key Class in developing an understanding of the evolution of cognitive abilities amongst amniotes. The study of similarities and differences in their cognition can inform about homologies and analogies of cognitive mechanisms across the amniotes [5]. Traditionally reptiles are considered to be limited in terms of their cognition [6], however, when tested in physiologically appropriate conditions, they show an impressive suite of cognitive abilities [e.g. $\left.7^{* *}, 8^{* *}, 9-11\right]$.

Reptiles are also interesting in the study of cognition because of their pattern of development. In birds and mammals, development takes place under stable conditions at high temperatures (though other sources of variation may influence the developmental environment [e.g. 12]). By contrast, reptiles develop normally at a range of temperatures, which fluctuate during incubation, and this affects the phenotype of the hatchling in terms of sex, growth rate, physiology, pigmentation, locomotion, and behaviour [for reviews see 13,14]. Therefore, these differing early environments may also be affecting the cognitive abilities of individuals [e.g. 15-18], which may have long-term fitness implications. The stable environments of avian or mammal development do not produce this range of phenotypic variability.

The study of reptiles is essential for understanding cognitive evolution, but this group also allows us to test fundamental hypotheses in the field of cognition which simply cannot be tested using mammals and birds. This review will summarise some key trends in the field, wherever possible we will present all relevant recent work, therefore, any imbalance in the species discussed is likely to reflect the paucity of knowledge in the area. We will then go on to outline some (but by no means all) areas in which the use of reptiles can change our understanding of the processes shaping cognition before finally discussing some future directions for the field.

\section{The impact of early environment on cognition}

Effects of incubation temperature 
Personality and cognitive performance appear to be linked within a species [19, 20]. Therefore, differences in personality are likely to play an important role in understanding individual differences observed in cognitive performance [20,21]. As such, it is essential to consider the impact of early environmental conditions on personality when considering its impact on cognition. Our recent work assessed the impact of incubation temperature on bearded dragons (Pogona vitticeps) in two classic personality tests - behaviour towards a novel object, and behaviour in a novel environment - tests were repeated after a few days and several months [22**]. Although incubation temperature had a short-term effect on the time spent in close proximity to a novel object, no long-term effect was observed $\left[22^{* *}\right]$. Hence, variation in incubation temperature within the normal range may impact the development of behavioural traits rather than long-term differences in personality. This in turn may influence cognitive performance over development, which could result in long-term differences in cognitive ability.

Incubation temperature outside the normal range appears to have an even greater effect on the behaviour of offspring. Holleley and colleagues [23] showed that incubation at a very high temperature $\left(\geq 32^{\circ} \mathrm{C}\right.$ ) produced bearded dragons that were genetically male but functionally female. These animals exhibited behavioural traits that appeared to be more male-like than genetic females. This included behaviours associated with personality, e.g. higher activity levels and faster emergence from a shelter [24*]. Though the mechanism underlying these differences is unknown, incubation at extreme ends of the temperature range produces offspring that behave in a manner that is different from individuals incubated within the normal temperature range; how this impacts fitness has yet to be studied [24*].

Anatomical differences have been shown in brain structure and neuron density under different incubation conditions; at hatching hot-incubated Eastern three-lined skinks (Bassiana duperreyi) have greater neuron density in parts of their medial cortex than their cooler incubated counterparts [15]. This is supported by behavioural data which shows that incubation environment impacts learning, with hot-incubated skinks of the same species performing better in operant learning tasks than coldincubated individuals [16-18]. By contrast, bearded dragons incubated in cooler conditions perform better in both an operant [Juntilla et al.,in prep] and a social learning task [Siviter et al.,submitted].

These studies suggest that differences in cognition are not based on absolute differences in temperature but are likely to reflect the environment in which the animals are hatching into. Responses to incubation temperature are likely to differ across species and habitat but a hotter incubation environment may result in offspring that are better adapted for survival in that temperature range $\left[22^{* *}\right]$. This is contemporary because habitats across the globe are being increasingly affected by environmental change, which creates novel challenges for the animals living there [25]. Reptiles are particularly interesting in this context because of their reliance on external sources of heat [26]. Whilst genetic adaptation can be slow, changes in behaviour may allow more rapid adaptation to the novel 
conditions [27]. Cognitive abilities are likely to be important in relation to this as they influence all aspects of decision making [28].

\section{Impact of Early Social Environment}

In contrast to mammals and birds, many reptile species provide limited or no parental care of eggs or their offspring [29]. This provides opportunities to control and manipulate the social environment during early stages of development so as to try to disentangle the impacts of social environments on cognition.

Traditionally, social interactions among eggs have been assumed to be limited as each egg is isolated by the shell. Embryonic communication is well known in birds [30] although its effects on subsequent social behaviour is unknown. In reptiles, embryonic communication has recently been reported [3133] and has been shown to influence behaviour post-hatching [34]. Hatchling water snakes (Natrix maura) from eggs incubated in isolation occupied a larger space, were more mobile and maintained less physical contact with conspecifics than counterparts incubated in a clutch where eggs were in contact [34].

The social environment post-hatching also seems to impact behaviour. Veiled chameleons (Chamaeleo calyptratus) reared in social isolation after hatching had a longer latency to catch prey, exhibited more submissive behaviour and different colouration from their socially-reared counterparts [35], although this has not yet been shown to impact cognition. In the only study of its kind, Riley and colleagues [36], reared the group living tree skink (Egernia striolata) in social isolation or in groups. Contrary to the prediction that those raised in a social environment would perform better in a spatial cognitive task, results revealed that social environment did not have an effect on learning in a biologically relevant spatial task [36]. It remains unknown whether these conditions would impact on performance of a social cognition task.

\section{Impact of early cue experience on spatial learning}

Much cognition research with Chelonia has investigated spatial navigation with the focus on the impressive long-range navigational feats of sea turtles [37-39] and short-range spatial learning in terrapins and tortoises [9, 40-43]. There is evidence for use of geomagnetic cues, visual cues and response-based strategies in these species [44].

The importance of early life experience on this ability has been examined in the loggerhead turtle (Caretta caretta), known to migrate the long distances across the Atlantic Ocean. Geomagnetic cues were manipulated during incubation, by placing magnets around the eggs, and was shown to impact 
on hatchling navigation. The hatchlings from the manipulated magnetic field swam in random directions, while the control group oriented towards the migratory route, suggesting that experimental magnetic fields during incubation can alter hatchlings ability to navigate using magnetic cues [45].

Recent field studies on translocated terrapins (Chrysemys picta) revealed that individuals under the age of four were able to follow paths of adults [46**] but this ability was lost in older individuals. This suggests that there may be a critical phase for learning. This may be particularly important in the context of changing environments.

\section{Future directions}

We have highlighted recent work investigating the impact of early environmental conditions on reptile cognition. Much remains unknown, however, we hope we have demonstrated how the use of reptiles can provide insight into exciting new cognitive questions. Future studies need to involve more species and to fill in the gaps in our understanding. In terms of the traditional comparative approach, three key directions are required, which are discussed below.

Development of model reptile species: Comparative investigations of reptile cognition tend to discuss findings relative to our understanding of similar processes in mammals and birds. However, lack of data prevents an effective and comprehensive comparison. Therefore, we need to systematically replicate classic studies conducted in mammals and birds with reptiles that we feel can be representative model species. One candidate for this would be the red-footed tortoise (Chelonoidis carbonaria), a species that has proved adept across a number of cognitive domains (Wilkinson \& Glass, submitted) including social [10, 47, 48], visual [7**, 49] and spatial cognition [9, 40-42].

Cognition across a range of reptile species: Generally, studies examine the cognitive abilities of one or two species and consider it in terms of what reptiles may be capable of. However, this approach would be unacceptable in mammals and birds and simply highlights the lack of knowledge for reptiles. In general, the choice of species used for this work lacks a systematic approach. Studies comparing the cognitive abilities of species selected in a systematic way, on the basis of phylogeny, would prove informative. Further, research on a wider variety of species from all four orders of reptiles would inevitably lead to a better understanding of phylogeny of cognition.

The impact of behavioral ecology on reptile cognition: Much work in comparative cognition uses the behavioural ecology of closely related species to predict differences in cognitive abilities [50]. This approach has proved extremely fruitful and should be harnessed in the field of reptile cognition. Some early research has revealed that feeding ecology impacts reversal but not spatial learning in congeneric lizards [51]. However, much more work which uses this approach with a wider range of ecological 
factors and over a greater variety of taxa are required.

\section{Understanding the adaptive value of cognition}

To understand the adaptive value of cognition, it is essential to develop biologically appropriate, hypothesis driven tests that can be run in the laboratory or the field. Field experiments will inherently have less control than those in laboratory settings, however, captive work cannot tell us about the selection pressures that operate under natural conditions. It is thus essential to integrate both laboratory and field work to better understanding of mechanisms and adaptation value of reptile cognition. This must be investigated in terms of the adaptive value to the animal, but also the impact that the cognitive processes may have on the ecosystem around it [ $\left.7^{* *}, 52\right]$.

\section{Conclusion}

In order to gain a fuller understanding of the evolution of cognition, it is essential to investigate the cognitive abilities of reptiles. To do this appropriately, we need to: i) conduct work with model species that are comparable to the vast amount of research undertaken with mammals and birds; ii) compare species that are of interest in terms of phylogeny; and iii) compare similar species that differ in terms of their behavioural ecology. In addition to fundamental comparative research, the use of reptiles can allow us to test critical hypotheses, such as the impact of early environment on cognitive abilities, in highly controlled environments which are difficult to investigate in mammals and birds. In conclusion, engaging with the study of "cold-blooded cognition" can only be informative and fascinating.

\section{Acknowledgements}

We would like to thank the cold-blooded cognition group at the University of Lincoln for discussions and two anonymous reviewers for their constructive comments. 


\section{References}

Papers of particular interest, published within the period of review, have been highlighted as:

* of special interest

** of outstanding interest

[1] Beach FA: The snark was a boojum. Am Psychol 1950, 5:115-124.

[2] Shettleworth SJ: The evolution of comparative cognition: Is the snark still a boojum? Behav Processes 2009, 80:210-217.

[3] Emery NJ, Clayton NS: The mentality of crows: Convergent evolution of intelligence in corvids and apes. Science 2004, 306:1903-1907.

[4] Emery NJ, Clayton NS: Evolution of the avian brain and intelligence. Curr Biol 2005, 15:R946-R950.

[5] Fitch WT, Huber L, Bugnyar T: Social cognition and the evolution of language: Constructing cognitive phylogenies. Neuron 2010, 65:795-814.

[6] Burghardt GM: Learning processes in reptiles. In Biology of the reptilia. Edited by Gans C, Tinkle DW. Academic Press; 1977:555-681.

[7] **Soldati F, Burman OHP, John EA, Pike TW, Wilkinson A: Long-term memory of relative reward values. Biol Lett 2017, 13:doi:10.1098/rsbl.2016.0853.

The study investigated long-term memory in reptile. The authors demonstrated that tortoises were able to remember the visual stimuli that represented different qualities and quantities of food for at least 18 months.

[8] **Kis A, Huber L, Wilkinson A: Social learning by imitation in a reptile (Pogona vitticeps). Anim Cogn 2015, 18:325-331.

This is the first study to demonstrate imitation in reptile, this is considered to be a cognitively complex behaviour and had previously been observed in only a few mammal and bird species. The authors show that bearded dragons are capable of copying a demonstrator to get access to food.

[9] Mueller-Paul J, Wilkinson A, Aust U, Steurer M, Hall G, Huber L: Touchscreem performance and knowledge transfer in the red-footed tortoise (Chelonoidis carbonaria). Behav Processes 2014, 106:187-192. 
[10] Wilkinson A, Huber L: Cold-blooded cognition: Reptilian cognitive abilities. In The oxford handbook of comparative evolutionary psychology. Edited by Shackelford TK, Vonk J. Oxford University Press; 2012:129-143.

[11] Leal M, Powell BJ: Behavioural flexibility and problem-solving in a tropical lizard. Biol Lett 2011, 8:28-30.

[12] Rokka K, Pihlaja M, Siitari H, Soulsbury CD: Sex-specific differences in offspring personalities across the laying order in magpies Pica pica. Behav Processes 2014, 107:7987.

[13] Booth DT: Influence of incubation temperature on hatchling phenotype in reptiles. Physiol Biochem Zool 2006, 79:274-281.

[14] Deeming DC: Post-hatching phenotypic effects of incubation in reptiles. In Reptilian incubation: Environment, evolution and behaviour. Edited by Deeming DC. Nottingham University Press; 2004:229-251.

[15] Amiel JJ, Bao S, Shine R: The effects of incubation temperature on the development of the cortical forebrain in a lizard. Anim Cogn 2017, 20:117-125.

[16] Amiel JJ, Lindstrom T, Shine R: Egg incubation effects generate positive correlations between size, speed and learning ability in young lizards. Anim Cogn 2014, 17:337-347.

[17] Amiel JJ, Shine R: Hotter nests produce smarter young lizards. Biol Lett 2012, 8:372-374.

[18] Clark BF, Amiel JJ, Shine R, Noble DWA, Whiting MJ: Colour discrimination and associative learning in hatchling lizards incubated at 'hot' and'cold' temperatures. Behav Ecol Sociobiol 2014, 68:239-247.

[19] Carazo P, Noble DWA, Chandrasoma D, Whiting MJ: Sex and boldness explain individual differences in spatial learning in a lizard. Proc R Soc B 2014, 281:doi:10.1098/rspb.2013.3275.

[20] Sih A, Del Giudice M: Linking behavioural syndromes and cognition: A behavioural ecology perspective. Philos Trans R Soc Lond B Biol Sci 2012, 367:2762-2772.

[21] Guenther A, Brust V, Dersen M, Trillmich F: Learning and personality types are related in cavies (Cavia aperea). J Comp Psychol 2014, 128:74-81. 
[22] **Siviter H, Deeming DC, Rosenberger J, Burman OHP, Moszuti SA, Wilkinson A: The impact of egg incubation temperature on the personality of oviparous reptiles. Anim Cogn 2017, 20:109-116.

This is the first study to investigate the effect that differences in incubation temperature (within the normal range) can have on hatchling personality and emphasises the importance of investigating long-term effects of early environment.

[23] Holleley CE, O’Meally D, Sarre SD, Marshall Graves JA, Ezaz T, Matsubara K, Azad B, Zhang $\mathrm{X}$, Georges A: Sex reversal triggers the rapid transition from genetic to temperaturedependent sex. Nature 2015, 523:79-82.

[24] * Li H, Holleley CE, Elphick M, Georges A, Shine R: The behavioural consequences of sex reversal in dragons. Proc $R$ Soc B 2016, 283:doi:10.1098/rspb.2016.0217.

The authors show that incubation temperatures at the extremes of the normal range ca have profound effects on offspring in terms of physiology and behaviour. Sex reversed females exhibited more male-like phenotypes compared to non sex reversed females.

[25] Mainwaring MC, Barber I, Deeming DC, Pike DA, Roznik EA, Hartley IR: Climate change and nesting behaviour in vertebrates : A review of the ecological threats and potential for adaptive responses. Biol Rev Camb Philos Soc 2016, doi:10.1111/brv.12317.

[26] Pough FH, Andrews RM, Crump ML, Savitzky AH, Wells KD, Brandley MC: Herpetology. Sinauer Associates, Inc.; 2015.

[27] Tuomainen U, Candolin U: Behavioural responses to human-induced environmental change. Biol Rev Camb Philos Soc 2011, 86:640-657.

[28] Greggor AL, Thornton A, Clayton NS: Neophobia is not only avoidance: Improving neophobia tests by combining cognition and ecology. Curr Opin Behav Sci 2015, 6:82-89.

[29] Balshine S: Patterns of parental care in vertebrates. In The evolution of parental care. Edited by Royle NJ, Smiseth PT, Kolliker M. Oxford University Press; 2012:62-80.

[30] Brua RB: Parent-embryo interactions. In Oxford ornithology series 13. Edited by Deeming DC. Oxford University Press; 2002:88-99.

[31] Aubret F, Blanvillain G, Bignon F, Kok PJR: Heartbeat, embryo communication and 
hatching synchrony in snake eggs. Sci Rep 2016, 6:doi:10.1038/srep23519.

[32] McGlashan JK, Loudon FK, Thompson MB, Spencer RJ: Hatching behavior of eastern longnecked turtles (Chelodina longicollis): The influence of asynchronous environments on embryonic heart rate and phenotype. Comp Biochem Physiol A Mol Integr Physiol 2015, 188:58-64.

[33] Vergne AL, Mathevon N: Crocodile egg sounds signal hatching time. Curr Biol 2008, 18:R513-514.

[34] Aubret F, Bignon F, Kok PJR, Blanvillain G: Only child syndrome in snakes: Eggs incubated alone produce asocial individuals. Sci Rep 2016, 6:doi:10.1038/srep35752.

[35] Ballen C, Shine R, Olsson M: Effects of early social isolation on the behaviour and performance of juvenile lizards, Chamaeleo calyptratus. Anim Behav 2014, 88:1-6.

[36] Riley JL, Noble DWA, Byrne RW, Whiting MJ: Does social environment influence learning ability in a family-living lizard? Anim Cogn 2016, doi:10.1007/s10071-016-1068-0.

[37] Godley BJ, Blumenthal JM, Broderick AC, Coyne MS, Godfrey MH, Hawkes LA, Witt MJ: Satellite tracking of sea turtles: Where have we been and where do we go next? Endanger Species Res 2008, 4:3-22.

[38] Lohmann KJ, Luschi P, Hays GC: Goal navigation and island-finding in sea turtles. $J$ Exp Mar Bio Ecol 2008, 356:83-95.

[39] Lohmann KJ, Cain SD, Dodge SA, Lohmann CM: Regional magnetic fields as navigational markers for sea turtles. Science 2001, 294:364-366.

[40] Mueller-Paul J, Wilkinson A, Hall G, Huber L: Radial-arm-maze behavior of the red-footed tortoise (Geochelone carbonaria). J Comp Psychol 2012, 126:305-317.

[41] Wilkinson A, Coward S, Hall G: Visual and response-based navigation in the tortoise (Geochelone carbonaria). Anim Cogn 2009, 12:779-787.

[42] Wilkinson A, Chan HM, Hall G: Spatial learning and memory in the tortoise (Geochelone carbonaria). J Comp Psychol 2007, 121:412-418. 
[43] Lopez JC, Gomez Y, Rodriguez F, Broglio C, Vargas JP, Salas C: Spatial learning in turtles. Anim Cogn 2001, 4:49-59.

[44] Mueller J, Wilkinson A, Hall G: Spatial cognition in reptiles. In Reptiles: Biology, behavior and Conservation. Edited by Baker KJ. Nova Science Publishers, Inc.; 2011:81-100.

[45] Fuxjager MJ, Davidoff KR, Mangiamele LA, Lohmann KJ: The geomagnetic environment in which sea turtle eggs incubate affects subsequent magnetic navigation behaviour of hatchlings. Proc R Soc B 2014, 281:doi:10.1098/rspb.2014.1218.

[46] **Roth TC II, Krochmal AR: The role of age-specific learning and experience for turtles navigating a changing landscape. Curr Biol 2015, 25:333-337.

This study suggest, for the first time, that there might be a critical period for learning in reptiles. This is also an excellent example of the benefits of combining both field and laboratory experiment for comprehensisve understanding of reptile cognition.

[47] Wilkinson A, Kuenstner K, Mueller J, Huber L: Social learning in a non-social reptile (Geochelone carbonaria). Biol Lett 2010, 6:614-616.

[48] Wilkinson A, Mandl I, Bugnyar T, Huber L: Gaze following in the red-footed tortoise (Geochelone carbonaria). Anim Cogn 2010, 13:765-769.

[49] Wilkinson A, Mueller-Paul J, Huber L: Picture-object recognition in the tortoise Chelonoidis carbonaria. Anim Cogn 2013, 16:99-107.

[50] MacLean EL, Merritt DJ, Brannon EM: Social complexity predicts transitive reasoning in prosimian primates. Anim Behav 2008, 76:479-486.

[51] Day LB, Crews D, Wilczynski W: Spatial and reversal learning in congeneric lizards with different foraging strategies. Anim Behav 1999, 57:393-407.

[52] John EA, Soldati F, Burman OHP, Wilkinson A, Pike TW: Plant ecology meets animal cognition: Impacts of animal memory on seed dispersal. Plant Ecol 2016, 217:1441-1456. 